\title{
植物对重金属耐性的分子生态机理
}

\author{
谭万能 ${ }^{2} 2$ 李志安 ${ }^{1 *}$ 邹 碧 ${ }^{1}$ \\ （1 中国科学院华南植物园，广州 510650) (2 中国科学院研究生院 北京 100039)
}

摘 要 植物适应重金属元素胁迫的机制包括阻止和控制重金属的吸收、体内鳌合解毒、体内区室化分隔以及代 谢平衡等。近年来, 随着分子生物学技术在生态学研究中的深入应用, 控制这些过程的分子生态机理逐渐被揭示 出来。菌根、根系分泌物以及细胞膜是控制重金属进入植物根系细胞的主要生理单元。外生菌根能显著提高寄主 植物的重金属耐性 根系分泌物通过改变根际 $\mathrm{pH}$ 、改变金属物质的氧化还原状态和形成络合物等机理减少植物对 重金属的吸收。目前, 控制菌根和根系分泌物重金属抗性的分子生态机理还不清楚。但细胞膜跨膜转运器已得到 深入研究 相关金属离子转运器被鉴定和分离,一些控制基因如铁锌控制运转相关蛋白 (ZIP)类、自然抵抗相关巨噬 细胞蛋白 (Nramp) 类、 $\mathrm{P}_{1 \mathrm{~B}}$-type ATPase 类基因已被发现和克隆。金属硫蛋白 (MTs)、植物螯合素 ( PCs)、有机酸及氨基 酸等是植物体内主要的螯合物质, 它们通过螯合作用固定金属离子, 降低其生物毒性或改变其移动性。与 MTs 合 成相关的 MT-like 基因已经被克隆,PCs 合成必需的植物螯合素合酶 (PCS)，即 $\gamma$-Glu-Cys 二肽转肽酶 ( $\gamma$-ECS) 的编 码基因已经被克隆 控制麦根酸合成的氨基酸尼克烟酰胺 (NA)在重金属耐性中的作用和分子机理也被揭示出来。 ATP 结合转运器 $(\mathrm{ABC})$ 和阳离子扩散促进器 ( $\mathrm{CDF}$ ) 是植物体内两种主要膜转运器, 通过它们和其它跨膜方式, 重金 属被分隔贮藏于液泡内。控制这些蛋白转运器合成的基因也已经被克隆，在植物中的表达证实其与重金属的体内 运输和平衡有关。热休克蛋白 (HSP) 等蛋白类物质的产生是一种重要的体内平衡机制, 其分子机理有待进一步研 究。重金属耐性植物在这些环节产生了相关响应基因或功能蛋白质, 分子克隆和转基因技术又使它们在污染治理 上得到了初步的应用。

关键词 重金属 分子机理 膜运转器 螯合物质 区室化 体内平衡

\section{MOLECULAR MECHANISMS OF PLANT TOLERANCE TO HEAVY METALS}

\author{
TAN Wan-Neng ${ }^{1}{ }^{2}$ LI Zhi-An ${ }^{*}$ and ZOU Bi ${ }^{1}$ \\ (1 South China Botanical Garden, Chinese Academy of Sciences , Guangzhou 510650 , China) \\ (2 Graduate School of Chinese Academy of Science, Beijing 10039 , China)
}

\begin{abstract}
Plants have evolved many adaptive mechanisms to cope with heavy metal stress , including governing uptake of heavy metal ions, detoxification by chelation, intracellular sequestration and cellular homeostasis to minimize the damage from exposure to nonessential metal ions. Mycorrhizae, root exudates and cellular membranes are three key factors that regulate heavy metal uptake. Ectomycorrhizae can significantly enhance the heavy metal tolerance of the host plant, and root exudates reduce the absorption of heavy metal ions by changing the $\mathrm{pH}$ and redox state of rhizsphere as well as chelation. However, the molecular mechanisms of these processes are not yet clear. The trans-membrane transporters have been fully researched and have been either identified or isolated. Many genes are involved, such as the ZIP (ZRT IRT related proteins) family , natural resistance associated macrophage proteins ( Nramp) and $\mathrm{P}_{1 \mathrm{~B}}$-type ATPase family were discovered and cloned in recent years. The primary chelators of heavy metals in plants are metallothioneins (MTs), phytochelatins ( $\mathrm{PCs})$, organic acids and amino acids. They alleviate the toxicity of metal ions in plants by chelation. MT-like genes encoding MTs and genes encoding $\gamma$-glutamyl-cysteine synthetase have been cloned. Gene expression in tested plants has demonstrated the role of genes in tolerance to heavy metals. Genes of phytochelatins synthetase (PCs) helping synthesis of PCs, i. e. $\gamma$-Glu-Cys, also have been cloned. The nicotianamine gene , an amino acid involved in biosynthesis of muginetic acids, has been shown to contribute to tolerance and hyperacumulation to heavy metals. ABC-type (ATP-binding cassette) transporters and CDF (cation diffusion facilitators) as well as other trans-membranes transporters help to sequester the heavy metals in vacuoles. The genes governing these transporters have been cloned and expressed in plants, which show to con-
\end{abstract}


tribute to heavy metal adjustment. Plants with heavy metal tolerance have developed pertinent genes or functional proteins in these aspects. In recent years, we have increased our understanding of the molecular mechanisms of plant heavy metal tolerance and have used plants in the remediation of heavy metal contamination. Understanding molecular mechanisms of plant heavy metal tolerance might be a key step for heavy metal remediation.

Key words Heavy metal, Molecular mechanisms , Transporters, Chelators, Sequestration , Intracellular metal homeostasis

随着人类活动的不断增加，全球土地重金属污 染越来越严重,它已成为危害人类自身健康的重大 因素, 因而, 近年来重金属污染治理成为国际科学界 研究的热点问题 (Chaney et al. , 1997)。目前, 借助 植物富集作用的治理技术得到广泛推崇, 并取得了 相当的进展, 主要是发现了一批具有重金属耐性和 超富集能力的植物, 如大量十字花科植物对锌的超 富集、蜈蚣草( Pteris vittata) 对砷的超富集、印度芥菜 (Brassica juncea) 对镉的超富集等 (Baker \& Brooks， 1989)。随着这些超富集植物的发现, 深入理解这些 植物对重金属元素的耐性及超富集能力变得越来越 重要, 特别是在分子水平上的研究工作, 可以为进一 步寻找其它超富集植物提供依据, 也为诱导性超富 集植物的出现 或通过分子克隆技术获得超富集植 物提供了基础，进而使得植物修复 (Phytoremediation) 这一经济有效的方式清除土壤重金属污染成为可 能。事实上 科学界在这一领域已开展了大量的工 作，并对重金属毒害的形成、某些植物的耐性、抗性 及相关的富集性的分子生态机理有了很好的理解。 研究发现，一些长期生活在重金属污染环境中的植 物逐渐适应进化出一系列忍耐和抵抗重金属毒害的 生理机制，包括重金属元素的控制吸收、体内螯合固 定、细胞内分隔、生化忍耐策略以及其它体内平衡机 制等(Clemens，2001)。近年来，作为污染生态学研 究的一个重要课题, 在重金属污染土地的恢复和治 理的生理生态学基础方面, 我国有不少综述性文章 介绍其进展, 但对上述分子水平的研究工作, 特别是 生理忍耐机制的分子控制机理方面却鲜见全面的报 道，对这一工作进行系统的总结，对我国重金属污染 治理的研究和实践具有极其重要的意义。本文主要 从近年来在分子水平上研究植物重金属耐性的机理 的几个方面进行总结, 同时展望其在重金属污染治 理中可能的应用。

\section{1 重金属离子吸收控制机理}

植物有 3 种重金属吸收策略——排斥 (Excluder)、指示 ( Indicator) 和富集 ( Accumulator) ( Baker，
1981 ）其中排斥和富集是植物适应重金属胁迫的两 种主要机制, 根系吸收和控制重金属进入植物体内 的机制多种多样, 其中, 菌根、根系分泌物以及细胞 膜是植物吸收和控制重金属的主要生理单元。

\section{1 菌根}

菌根 (Mycorrhiza) 特别是外生菌根 (Ectomycorrhizas) 在植物重金属耐性中起着较为重要的作用, 在重金属污染的土壤中具有耐性的外生菌根能显著 提高寄主植物的重金属耐性 (Galli et al., 1994 ; 赵 中秋等, 2003)。菌根对植物重金属耐性有如下几种 主要生理机制 ( Jentschke \& Goldbold，2000) :菌丝鞘 (Hyphal sheath)的选择吸收控制其进入质外体，通过 增加菌丝鞘的疏水性减少重金属接近质外体的几 率，吸收至外生菌丝体，分泌螯合物质控制重金属的 移动性, 通过改变营养物质吸收的速度和量改变根 系 $\mathrm{pH}$ 值，以及分泌激素改变代谢过程减缓毒害等。 在外生和内生菌根真菌中, 重金属能被束缚在细胞 壁的组成成分中，如纤维素衍生物、黑色素及几丁质 (Galli et al. , 1994)。目前，控制菌根重金属耐性的 分子机理并不清楚, 相关研究也较少。最近 Vallino 等(2005) 研究了欧洲越桔 (Vaccinium myrtillus) 的菌 根树粉孢 (Oidiodendron maius) 耐 Zn 的分子机理, 利 用 $\mathrm{Zn}$ 处理的树粉狍的 mRNA 构建 cDNA 库, 然后利 用表达序列标记的方法分离鉴定出 76 个新的基因， 他们利用反转 northern-blot 杂交表达后得到 16 个与 $\mathrm{Zn}$ 抗性有关的基因, 并且初步探讨了每一个基因在 提高树粉孢抗性的可能作用。但是这些基因是不是 在真菌中普遍存在，其控制真菌表达重金属抗性的 机制是否一致仍需进一步研究。

\section{2 根系分泌物}

根系分泌物 (Root exudation) 在植物控制重金属 进入植物体中也起着重要的作用, 根系分泌物能够 减少植物对毒害重金属的吸收或者中止毒害金属物 质进入植物体。植物根系分泌物种类繁多、成分复 杂, 其中研究较多的是有机酸、氨基酸和糖类等可溶 性的有机小分子及高分子, 以及不溶性的粘胶类物 质。根系分泌物控制重金属吸收的机制包括改变根 
际 $\mathrm{pH}$, 改变金属物质的氧化还原状态, 与重金属络 合以及和根际微生物产生根际效应等 (赵中秋等, 2003)。其中根系有机酸物质对 $\mathrm{Al}$ 的耐性的影响研 究较为清楚, 研究表明菾麦 (Fagopyrum mill) 根系分 泌的草酸能够络合 $\mathrm{Al}$, 并且将形成的无毒的草酸铝 盐累积在叶片中 (Ma et al. , 1997a)。Salt (2001) 利 用 Ni 处理遏蓝菜属( Thlaspi) 的 T. arvense 时也发现 根系组氨酸和柠檬酸增加。不同的络合物和不同金 属之间的作用不一样, 例如有研究表明,玉米 (Zea mays ) 分泌物对 $\mathrm{Pb} 、 \mathrm{Cu} 、 \mathrm{Cd}$ 存在络合作用，但它只提 高 $\mathrm{Cu} 、 \mathrm{Mn}$ 的溶解性, 对 $\mathrm{Cd}$ 的溶解度没有影响 (Mench \& Martin，1991)。根系在重金属胁迫的时候 分泌有机酸等物质的控制机理不是很清楚, 研究发 现在小麦( Triticum aestivum) 和玉米中根系分泌物的 产生可能与 $\mathrm{Al}$ 激活的细胞膜上阴离子通道有关 ( Ma et al . ,2001)。

另一种研究得比较详细的根系分“必物是禾本科 植物铁载体 (Phyto-siderophores，PS)(曹慧等,2002)， 即麦根酸类物质 (Muginetic acids, MAs) ,包括麦根酸 (Muginetic acid，MA)、2'-脱氧麦根酸 ( $2^{\prime}$-deoxymuginetic acid，DMA)、3-表羟基麦根酸 (3-epihydroxymuginetic acid, epi-HMA)、3-表羟基 $2^{\prime}$-脱氧麦根酸 (3epihydroxy 2'-deoxymuginetic acid , epi-HDMA)，不同的 禾本科植物都会分泌其特定的麦根酸类物质, 在缺 铁状态下能显著提高铁的利用率，同时可能控制其 它重金属离子的吸收 (Curie \& Briat ,2003)。PS 的生 物合成途径已经基本上揭示清楚，第一步是非蛋白 氨基酸尼克烟酰胺 (Nicotianamine, NA)的合成; 第二 步也是关键的一步是尼克烟酰胺氨基转移酶 ( Nicotianamine aminotransferase, NAAT) 催化一个氨基酸残 基和 NA 结合形成 $2^{\prime}$-脱氧麦根酸, 即其它麦根酸类 物质的前体。在大麦 (Hordeum vulgare) 中发现了两 种控制 NAAT 合成的基因 NaatA, NaatB(Takahashi et $a l$. , 1999) ,在水稻(Oryza sativa)中表达发现它们和 铁胁迫相关 (Takahashi et al . , 2001)。2'-脱氧麦根 酸经过羟基化作用 (Hydroxylations) 形成其它的麦根 酸类物质，两种控制该过程的 $\mathrm{cDNA}$ 物质，缺铁胁迫 特异克隆 ( Iron deficiency-specific clone, Ids) Ids2 和 $I d s 3$ 已经被克隆出来 (Nakanishi et al . , 2000) , 其中 $I d s 2$ 可能和 MA 转化形成 epi-HMA, DMA 转化形成 epi-HDMA 有关, Ids3 可能和 DMA 转化形成 MA 有 关。

\section{3 细胞膜}

重金属进入植物根系细胞必需跨过根系细胞的
细胞膜 细胞膜的吸收或抑制吸收成为植物适应重 金属胁迫的重要机制之一。近年来, 利用分子生物 学手段研究植物细胞膜吸收金属离子已取得了显著 的进展, 相关金属离子转运器陆续被鉴定分离。通 过对酿酒酵母 (Saccharomyces cerevisiae) 的吸收缺陷 突变型的修复的研究，一系列 cDNA 编码的金属离 子转运器相继被克隆。比如拟南芥 (Arabidopsis thaliana) 铜转运器 (Copper transpoter) 基因 COPT1 能 修复酿酒酵母的高亲合性 $\mathrm{Cu}$ 吸收缺陷的突变型 ctrl-3 的缺陷症状, 被认为可能是 $\mathrm{Cu}$ 的转运器 (Kampfenkel et al .,1995)。

铁离子控制转运器 (Iron regulated transporter, IRT) 基因 IRTI 较早在拟南芥中被克隆, 它能修复酿 酒酵母的 $\mathrm{fet} 3 / \mathrm{fet} 4$ 突变体的缺陷症状。IRTI 表达 的酵母菌表现出很强的 $\mathrm{Fe}^{2+}$ 吸收能力, 而对 $\mathrm{Fe}^{3+}$ 则 几乎完全不亲合。除了 $\mathrm{Fe}^{2+}, I R T 1$ 对 $\mathrm{Cd}^{2+} 、 \mathrm{Co}^{2+}$ 、 $\mathrm{Mn}^{2+}$ 以及 $\mathrm{Zn}^{2+}$ 等有较低亲合度的吸收运转能力 (Eide et al. , 1996)。它在 Fe 缺乏的植物根系中得 到很好的表达, 相反, 在 $\mathrm{Fe}$ 过量的时候其表达会受 到抑制，从而使植物能够排除根系细胞原生质中多 余的 $\mathrm{Fe}$ 离子。

IRTI 的克隆使得一些编码铁锌离子控制转运 器相关蛋白 (ZRT IRT related Protein, ZIP)类的基因 相继被克隆出来。锌离子控制转运器 (Zinc regulated transporter, ZRT) 基因 ZRT1 和 ZRT2 通过和 IRT1 的 相似序列测定在酵母菌中得到鉴定，它们分别是 $\mathrm{Zn}$ 的高亲合和低亲合转运器 (Zhao \& Eide, 1996a, 1996b)。拟南芥中也克隆了 ZIP1、ZIP2、ZIP3、 $Z I P 4$ 其中 $Z I P 1-3$ 能运输 $\mathrm{Zn}^{2+} 、 \mathrm{Cd}^{2+}$ 和 $\mathrm{Cu}^{2+}$, 还能 以较低的亲合度运输 $\mathrm{Mn}^{2+}$ 。在 $\mathrm{Zn}^{2+}$ 缺乏的植物根 系中 $Z I P 1$ 和 ZIP3 能得到很好的表达, 同样在 $\mathrm{Zn}^{2+}$ 充足或者过量的时候其表达会受到抑制 (Grotz et al. 1998)。遏蓝菜属的 Thlaspi caerulescens 中也发 现了一种与 ZIP4 相似的锌运转器 (Zinc-transporter, ZNT) 基因 ZNT1 (Pence et al. 2000) ,进一步证明它 们可能与 $\mathrm{Zn}^{2+}$ 吸收密切相关。van der Zaal 等 (1999) 在拟南芥中克隆到一种与 ZNT1 类似的 ZATI ( Zn transporter of Arabidopsis thaliana ) 基因，这种基因的表 达不受 $\mathrm{Zn}^{2+}$ 控制，它在拟南芥中的超量表达被证实 和 $\mathrm{Zn}^{2+}$ 抗性有关。加上它和 ZNT1 的类似, ZAT 可 能是一种和根系主动外排 $\mathrm{Zn}^{2+}$ 有关的 $\mathrm{Zn}^{2+}$ 排出蛋 白(Efflux protein)。

另一类重要的金属离子运转器是自然抵抗相关 巨噬细胞蛋白 (Natural resistance associated macrophage 
proteins , Nramp)类。Nramp 基因最初是在哺乳动物 和酵母菌中发现的, 随后植物中也发现了其同源序 列并得到克隆。水稻中的 3 个 Nramp 基因 $O s$ Nramp1、OsNramp2 和 OsNramp3 以及拟南芥中的 5 个 Nramp 基因 AtNramp1、AtNramp2、AtNramp3、AtNramp4 和 AtNramp5 被相继克隆。其中 AtNrampl 能修复 $\mathrm{fet} 3 / \mathrm{fet} 4$ 缺陷型的缺 $\mathrm{Fe}$ 症状, 并且在 $\mathrm{Fe}$ 缺乏的时候 能够超量表达, 可能与 $\mathrm{Fe}$ 的吸收和体内平衡有关 (Curie et al.,2000)。AtNramp3 还可能与 $\mathrm{Cd}^{2+}$ 的吸 收有关。AtNramp1、AtNramp2 和 AtNramp3 在酵母菌 中的单独表达证明他们与 $\mathrm{Mn}^{2+}$ 吸收有关。这些转 运器在金属离子浓度增加时表达会受到抑制 (Thomine et al. ,2000)。

P-type ATPase 是一种通过水解 ATP 进行跨膜运 输的运转蛋白, 根据其底物特异性 (Substrate specificity) 可以分为 5 类, 其中 $\mathrm{P}_{1 \mathrm{~B}}$-type ATPase 是与多种 重金属离子如 $\mathrm{Cd}^{2+} 、 \mathrm{Cu}^{2+} 、 \mathrm{Cu}^{+}$和 $\mathrm{Zn}^{2+}$ 等跨膜运输 有关的运转器, 它也被称作重金属 ATP 酶 (Heavy metal ATPases, HMA)。几种不同的 HMA 基因最近 相继被报道, 其中拟南芥中克隆的 HMA2 是 $\mathrm{Zn}^{2+}$ 和 $\mathrm{Cd}^{2+}$ 的高亲合转运体，被证明是植物向体外排出 $\mathrm{Zn}^{2+}$ 的控制单元之一 ( Eren \& Argüello，2004)。 HMA4 与 $\mathrm{Zn}$ 的体内平衡以及 Cd 耐性有关 (Bernard et al. 2004), 同时它通过向胞外主动排出 (Active efflux) 过量的重金属离子提高 $\mathrm{Zn} 、 \mathrm{Cd}$ 和 $\mathrm{Pb}$ 的耐性 (Papoyan \& Kochian, 2004)。研究表明其结合和运 输重金属离子的能力与 $\mathrm{N}$ 末端的氨基酸残基和 $\mathrm{C}$ 末端的氨基酸延长结构有关(Verret et al .,2005)。

\section{2 重金属在植物体内的螯合机理}

重金属离子通过根系运转器以及其它途径进入 植物细胞后，通过螯合作用固定金属离子或者降低 其生物毒性是植物对细胞内重金属解毒的主要方 式。植物中已经发现的螯合物质主要有金属硫蛋白 (Metallothioneins, MTs)、植物螯合素 (Phytochelatins， $P C s$ )、有机酸 (Organic acid) 以及氨基酸 (Amino acid)。

2.1 金属硫蛋白 (MTs)

MTs 最先是在马肾中发现的一种与 Cd 解毒相 关的金属结合蛋白。它是一种普遍存在的低分子量 $(6 \sim 7 \mathrm{kD})$ 、富半胱氨酸、能被金属诱导的金属结合 蛋白，其合成受基因控制。MTs 一般包含两个富半 胱氨酸的金属结合域,形成以硫醇键结合的哑铃状 金属蛋白。MTs 按其 Cys 残基的排列方式分为两 类 :Class I MTs 包含 20 个高度保守的 Cys 残基 ,与马
肾中 MT 有极大的相似性, 多见于脊椎动物; Class II MTs 不包含严格的 Cys 序列, 多见于植物、真菌和无 脊椎动物。 PCs 曾经被认为是 Class III MTs (Cobbett \& Goldsbrough , 2002)。

植物中第一个被分离出来的 MTs 是小麦 EC (Early Cys labeled)蛋白，它是从成熟胚胎里面分离 出来的, 表现为 $\mathrm{Zn}^{2}$ 结合 (Lane et al , 1987)。随后 大约有 50 余种编码 MT 的 MT-like 基因在不同的植 物中被发现（Rauser，1999），根据氨基酸序列的不 同，这些编码 Class II MTs 的 MT-like 基因又可以分 为 Type $1-4$ 四类, 前 3 种都有两个富半胱氨酸的域 ( $\mathrm{C}$ 末端和 $\mathrm{N}$ 末端), Type 4 则有 3 个富半胱氨酸的 域, 主要以 Cys-Xaa-Cys 形式存在 (Cobbett \& Goldsbrough，2002)。不同的 MT-like 基因在植物体内表 达部位不一样, 比如 Garcia-Hernandez 等 (1998) 利用 RT-PCR 和原位杂交技术研究了拟南芥 MTla 和 $M T 2 a$ 基因，他们发现在拟南芥根系成熟区和叶表毛 状体中均有两种基因的 mRNA 物质, 同时 MTla 在 维管组织和叶肉细胞中也有表达, 而 $\mathrm{Cu}$ 诱导的 $M T 2 a$ 表达被限制在子叶中。

MTs 在植物体内参与重金属解毒和体内平衡也 已经得到证实。在酿酒酵母的 $\mathrm{Cu}$ 敏感突变体 cupl 中表达拟南芥 MT1 和 MT2 后其敏感症状消失 (Zhou \& Goldsbrough , 1994) ;Ma 等(1997b) 从紫羊茅( Festuca rubra cv. Merlin)中克隆出 $m c M T 1$ 基因 将其在酵 母菌中表达也得到了类似的结果;两个试验同时也 表现出 $\mathrm{Cd}^{2+}$ 抗性。MT2 还能部分缓解聚球藻属 (Synechococcus) $\mathrm{Zn}^{2+}$ 的突变体 $s m t A$, 因为缺乏 MT 表 现出的 $\mathrm{Zn}^{2+}$ 超敏感症状 (Robinson et al. , 1996)。拟 南芥中编码 MT 的基因 AtMT2 $a$ 和 AtMT3 与植物细 胞 $\mathrm{Cd}$ 抗性有关, 试验表明 $\mathrm{Cd}$ 抗性提高与 $A t M T 2 a$ 和 AtMT3 减少了细胞表面活性氧物质有关 (Lee et al. 2004)。

由于有氧条件下不稳定以及表达水平和表达部 位的 RNA 水平相关等原因, 导致迄今为止 MT 研究 中蛋白质数据仍旧缺乏 ( Cobbett \& Goldsbrough , 2002），进一步了解植物重金属耐性中 MT-like 基因 的作用较为困难。除了最早分离出来小麦 EC 蛋白 和最近分离出来的拟南芥 MT1 和 MT2 蛋白以外, 从 植物组织中提 MT 蛋白均未成功, 只有 EC 蛋白与重 金属结合得到证明。

\section{2 植物螯合素 $\left(P C_{s}\right)$}

植物对重金属胁迫的重要适应机制之一是产生 植物螯合素。植物螯合素是一系列具有 ( $\gamma$-Glu- 
Cys) ${ }_{\mathrm{n}}$-Gly $(n>1$,多为 $2 \sim 11$ ) 结构的多肽 (Grill et $a l ., 1985)$ 。植物螯合素在很多高等植物以及真菌、 藻类中都有发现。在植物中, 还存在以其它氨基酸 为 C-末端的 PCs 分子, 包括 ( $\gamma$-Glu-Cys ) ${ }_{n}-\beta-A l a, ~(\gamma-$ Glu-Cys) ${ }_{n}$-Ser 和 ( $\gamma$-Glu-Cys $)_{n}$-Glu 等形式 (Rauser , 1999)。PCs 在植物重金属耐性中的作用 (如 Cd 抗 性、重金属解毒以及维持体内金属离子平衡等)已经 有较为详尽的报道 (Rauser, 1995 ; Cobbett，2000)。

PCs 是通过谷胱甘肽 (Glutathione, GSH) 生物合 成的，GSH 合成抑制剂可使植物 PCs 合成受到抑制 或者表现为对 Cd 超敏感(Steffens et al . 1986)，相 反, 最近 Freeman 等 (2004) 发现丝氨酸乙酰转移酶 (Serine acetyltransferase, SAT) 活性升高引起的 GSH 浓度升高, 则提高了遏蓝菜 $\mathrm{Ni}$ 超富集植物对 $\mathrm{Ni}$ 富 集引起的活性氧胁迫的忍耐程度。Grill 等(1989)通 过膀胱麦瓶草 (Silene cucubalis) 体外培养证实 GSH 是 PCs 合成前体, 并且发现了催化 PCs 合成的植物 螯合素合酶 (Phytochelatin synthase, PCS) ,即 $\gamma$-GluCys 二 肽转肽酶 ( $\gamma$-glutamyl cysteine dipeptidyl transpeptidase, $\gamma$-ECS)。PCs 的合成包括以下两个步 骤:1)谷胱甘肽的生物合成。谷胱甘肽的合成包括 以下两个过程: $\gamma-$ ECS 催化谷氨酸和半胱氨酸合成 谷氨酰半胱氨酸二肽和谷胱甘肽合成酶 (Glutathione synthetase, GS) 催化甘氨酸与 $\gamma$-谷氨酰半胱氨酸二 肽合成单谷胱甘肽。2) PCs 的合成 (Cobbett,2000)。 编码 PCS 合成的基因已经被克隆, 通过对拟南芥 Cd 敏感突变型的定位克隆得到一个编码 PCs 合酶的基 因 $C A D 1$ (后被命名为 $A t P C S 1)$, 在大肠杆菌 $(E s$ cherichia coli)中表达证明其表达产物是 PCS ( Ha et $a l$. , 1999)。与此同时, 通过对 Cd 耐性的 cDNA 库 的節选克隆，小麦和拟南芥中控制 PCS 合成的基因 TaPCS1 和 AtPCS1 也分别被发现 (Clemens et al., 1999)，裂殖酵母菌 (Schizosaccharomyces pombe) 中也 发现了类似的 SpPCS1。除此而外, Cobbett 和 Goldsbrough(2002)利用拟南芥 $c a d 1$ 突变体进行 Cd 处理 时意外得到另一种基因 $A t P C S 2$, 它在植物体内表达 远远低于 AtPCS1 ,它在植物重金属耐性的作用尚待 进一步研究。Gong 等 (2003) 应用乙醇脱氢酶 (Alcohol dehydrogenase, Adh) 启动子将小麦 TaPCS1 转基 因表达至拟南芥 $c a d 1-3$ 缺陷型, 结果显示 $T a P C S 1$ 基因抑制了 $c a d 1-3$ 对重金属的敏感性。同时该试 验证明 PCs 不只是一种胞内的耐性机制，它也可以 从根部向茎杆运输, 从而增加了 Cd 从根部向茎杆的 长距离运输, 减少了根部镉的浓度。

\section{3 有机酸和氨基酸}

有机酸和氨基酸在重金属而性中的作用在前述 的根系分泌物中已有提及, 已有多种有机酸如柠檬 酸, 苹果酸以及草酸等被证实参与植物体内多种过 程, 包括不同重金属的耐性、木质部金属运输以及液 泡区室化重金属(Rauser，1999)。

有机酸与重金属耐性相关的有力证据仍然很缺 乏, 金属胁迫和有机酸产生之间很少发现线性相关 关系。这与遗传数据的缺乏、常规分析手段如 X-射 线吸收光谱得到的数据被限制在金属离子的配位环 境等因素有关。而且金属配位体的产生与区室化作 用,其它离子的可利用性以及 $\mathrm{pH}$ 等因素有关 (Clemens，2001)，因此二者之间的对应关系很难证 实。在庭荠属 (Alyssum) 的 $\mathrm{Ni}$ 超富集植物 A. lesbiacum 中发现经 $\mathrm{Ni}$ 处理后木质部液流中组氨酸浓度 提高了 36 倍, 而非超富集的 A. montanum 中则没有 发现这种组氨酸效应 (Histidine response),加入组氨 酸过后 其 $\mathrm{Ni}$ 耐性增加(Krämer et al .,1996)。但是 最近研究表明这并不是普遍存在的机制, 在另一种 $\mathrm{Ni}$ 超富集植物遏蓝菜属的 Thlaspi goesingense 中并没 有发现这种现象 (Persans et al. , 1999) , 其分子机理 也有待进一步的研究。

另一个研究较为详细的氨基酸是尼克烟酰胺, 它是三分子的 S-腺苷甲硫氨酸 (S-adenosyl methionine, SAM) , 是植物铁载体麦根酸的前体。它是植物 体内 $\mathrm{Fe}^{2+}$ 和其它二价金属的配位体 (Stephan \& Scholz, 1993)。SAM 合成 NA 的过程受尼克烟酰胺 合酶(Nicotianamine synthase, NAS) 控制, 控制 NAS 合 成的 cDNA 也已经被克隆出来 (Higuchi et al. , 1999)。在玉米中分离出 3 种 NAS 基因 根据它们的 表达类型和胞内分布可以分为两类。Z ZmNAS1 和 $Z m N A S 2$ 在植物缺铁的时候表达, 而 ZmNAS3 在铁过 量的时候表达; ZmNAS1 和 ZmNAS2 的叶绿素苂光融 合蛋白位于表皮细胞胞质呈点状分布, 而 $Z m N A S 3$ 则贯穿整个胞质 (Mizuno et al. 2003)。拟南芥 NAS 在转基因烟草中异位表达发现能够增加成体植物口十 片中铁含量，提高铁缺乏状况下铁的利用率增加植 物体内 $\mathrm{Zn}$ 和 $\mathrm{Mn}$ 的累积, 同时还提高了对

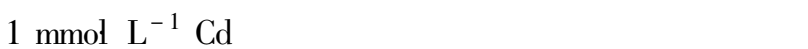
平衡机制中有重要的作用(Douchkov et al. ,2005)。

\section{3 重金属体内分隔机理}

重金属进入植物体后, 植物必需将其从细胞质 中排出以避免重金属毒害, 植物去除重金属的主要 
机制有排出体外和细胞内区室化。研究表明液泡是 植物和酵母中主要的分离悾藏重金属的部位, 在裂 殖酵母菌中 ,PC-Cd 复合体被运输到液泡中 ( Ortiz et $a l ., 1995)$ 燕麦 (Avena sativa) 中也发现了类似的现 象(Salt \& Rauser, 1995)。通过对麦瓶草 (Silene vulgaris) 的 Zn 耐性和敏感品种液泡膜小囊的分离, 发 现耐性品种 Zn 通过液泡膜进入小囊是敏感品种的 2.5 倍, 从而证实液泡膜在重金属离子区室化分隔 中起着重要作用(Verkleij et al. , 1998) , 进一步研究 证明液泡膜的重金属运输能力和一系列膜运转器有 关, 其中 ATP 结合盒 (ATP-binding cassette, ABC) 转 运器和阳离子扩散促进器 (Cation diffusion facilitator, $\mathrm{CDF}$ ) 是两种主要类型的膜转运器。

\subsection{ABC 型转运器}

$\mathrm{ABC}$ 型转运器是一种种类繁多、分布广泛、运转 物质类型多样的跨膜转运器。根据其系统发生途径 和结构特征的不同, $\mathrm{ABC}$ 基因被分为很多种类，由于 历史原因它们通常和药品抗性联系在一起, 比如主 要的 3 个类型命名均与药品抗性有关:多向耐药性 (Pleiotropic drug resistance, PDR)、多药耐药性 ( Multidrug resistance, MDR) 和多药耐药相关蛋白 (Multidrug resistance-associated protein, MRP)。其构造包 括两个主要的结构单元:一个疏水跨膜域 (Transmembrane domain, TMD) 和一个核苷酸结合域 ( Nucleotide-binding domain, NBD) (Jasinski et al . ,2003)。

植物中较早发现的液泡膜 ABC 转运器是 HMT1 (Heavy metal tolerance) ,HMT1 是一种和低分子量 PCCd 复合体 ( Low molecular weight, LMW) 向液泡中转 运形成高分子量复合体 (High molecular weight, HMW) 有关的蛋白, 它能修复裂殖酵母菌中一个不 能产生 HMW 的突变型的胁迫症状 (Ortiz et al. , 1992)。HMT1 位于液泡膜上, 主要调节依靠 MgATP 提供能量、能被钒酸盐抑制的 PC-Cd 复合体和脱辅 植物螯合素 (apo-PC) 的跨膜运输 (Ortiz et al. , 1995)。燕麦中也发现了类似的依靠 MgATP 提供能 量、钒酸盐敏感的运转机制 (Salt \& Rauser, 1995) 表 明 HMT1 运转是植物和酵母菌中重金属分隔的一种 重要机制。

在酿酒酵母中发现的另一个与 $\mathrm{Cd}^{2+}$ 耐性有关 的 $\mathrm{ABC}$ 型转运器是酵母镉基因 (Yeast cadmium factor, YCF1) ,它是一种 MRP 型 $\mathrm{ABC}$ 运转器, 在细胞中 起谷胱甘肽-S 共轭泉 (Glutathione S-conjugate pump) 作用，它通过和 bis (glutathiona-to) Cd 结合将其运输 至液泡从而达到分隔 (Li et al . , 1997)。在酿酒酵
母缺陷型突变体 DTY167 中表达发现它显著提高其 对 $\mathrm{Pb}$ 和 $\mathrm{Cd}$ 的耐性，同时也增加了对这两种金属的 体内累积 (Song et al. , 2003)。拟南芥中也发现了 MRP 相似的序列（Lu et al，，1997,1998) , 目前只有 $A t M R P 2$ 在液泡膜运转中的作用被证实,在解毒过 程中重金属离子和 GSH、Glc 以及葡萄糖苷酸等结 合形成亲水性化合物通过 $A t M R P 2$ 运输至液泡。

\section{2 阳离子扩散促进器}

$\mathrm{CDF}$ 是一类与植物和其它有机体重金属耐性 (排出体外或者胞内分隔) 有关的蛋白, 它们具有如 下特征:1) 特有的 N-末端信号序列 ( Signature sequence) 2) 具有 6 个跨膜域 ( Transmembrane spanning domain）3）有一个阳离子排出域 (Cation efflux domain）; )大多数真核生物中具有一个胞内富组氨酸 的域 (His-rich domain) ,而原核生物中没有 (Paulsen \& Saier , 1997)。

$\mathrm{CDF}$ 与植物内多种重金属离子的耐性有关, 其 中研究较多的是 $\mathrm{Zn}^{2+}$ 和 $\mathrm{Cd}^{2+}$,例如前面提及的拟南 芥 ZAT1 该基因也被称作 AtMTPs ,在表达该基因的 植物中表现出 $Z n$ 耐性增加, 在溶液培养中发现根部 $\mathrm{Zn}$ 累积增加而茎干中和对照区别不大, 因此该基因 的表达可能和 Zn 的分隔有关 (van der Zaal et al. , 1999) ,但是它在液泡膜的定位仍待研究。Blo $\beta$ 等 (2002)在大肠杆菌中表达 ZAT1 并利用提纯的脂蛋 白研究 $\mathrm{Zn}^{2}$ 转运结果发现 $Z A T 1$ 蛋白运转 Zn 跨膜 运输是依靠 Zn 浓度梯度而不是质子梯度。大肠杆 菌中另一种 $\mathrm{CDF}$ 基因 $Z n t A$ 在拟南芥中表达发现， 它位于细胞膜从而提高了植物对铅和镉的耐性。检 测出其茎杆中的铅和镉含量降低, 并且表达该基因 的拟南芥原生质体较之野生型有较少的镉累积和较 快的镉释放速度。说明这种细胞运转基因可以在植 物体中得到有效表达, 并且可能在重金属污染的植 物治理中起重要的作用(Lee et al. , 2003)。

Persans 等(2001)在 Ni 超富集植物遏蓝菜属的 Thlaspi goesingense 中分离出了一种编码金属忍耐蛋 白( Thlaspi goesingense metal tolerance protein)的基因 $T g M T P s$, 研究表明它能修复酵母菌 (Schizosaccharo myces cerevisiae) COT1 (Cobalt tolerance) 和 ZRC1 (Zinc resistance conferring) 缺陷型突变体的缺陷症状, COT1 和 ZRC1 是位于酵母菌液泡膜上与 $\mathrm{Co}^{2+}$ 和 $\mathrm{Zn}^{2+}$ 耐 性有关的 CDF 蛋白 (Li \& Kaplan, 1998)。TgMTP 可 能也与金属离子向液泡中转移有关, 但是其机理有 待进一步研究。Delhaize 等 (2003) 在笔花豆属 ( Stylosanthes) 的 $S$. hamata 中克隆了与 $\mathrm{Mn}^{2+}$ 耐性有关、 
编码 CDF 蛋白的 cDNA ShMTP1, 它们主要和 Mn 的 胞内分隔有关, ShMTP1 在酵母菌多种突变体上表 达后发现它是胞内细胞器上的 $\mathrm{H}^{+} / \mathrm{Mn}^{2+}$ 反转运器, ShMTP1 在拟南芥中表达也表现为胞内分隔,在拟 南芥中它与绿色苂光蛋白在液泡膜上融合, 作者认 为至少 ShMTP1 是一种编码和 $\mathrm{Mn}^{2+}$ 耐性相关 CDF 的基因。

\section{3 其它的离子转运器}

除了通过以上两种主要的运转器进入液泡外， 还有包括前述的根系细胞膜跨膜运转器的其它的运 转途径和运转途径存在。例如在裂殖酵母菌液泡中 形成 HMW 的过程中, 除了与 $\mathrm{S}$ 结合以外, 还有游离 的 $\mathrm{Cd}^{2+}$ 与之结合 (Rauser, 1995) ,研究表明这些 $\mathrm{Cd}^{2}+$ 可能是通过液泡膜上的 $\mathrm{Cd}^{2+} / \mathrm{H}^{+}$反转运器进 入液泡的。Salt 和 Wagner (1993) 在燕麦根系细胞中 发现了这种转运器, 它和液泡 $\mathrm{Ca}^{2+} / \mathrm{H}^{+}$反转运器 (Vacuolar $\mathrm{H}^{+} / \mathrm{Ca}^{2+}$ exchanger, VCX) 分子结构相类 似。编码 $\mathrm{Ca}^{2+} / \mathrm{H}^{+}$的钙交换器 CAX (Calcium exchangers) 基因已经被克隆, 拟南芥 CAX1、CAX2 能 够修复酵母菌 $v c x 1$ pmcl (Plasma membrane $\mathrm{Ca}^{2+}$ ATPases)双重缺陷型的 $\mathrm{Ca}^{2+}$ 吸收缺乏症状,在表达有 二者的酵母菌突变体中能检测到依赖 $\mathrm{pH}$ 的 $\mathrm{Ca}^{2}+$ 吸 收,而对照中没有。二者和 $\mathrm{Ca}^{2+}$ 的亲和程度不一 样 其中 $C A X 2$ 的 $\mathrm{Km}$ 值可能远远大于 $\mathrm{Ca}^{2+}$ 吸收的 需要, 因此它被推测与 $\mathrm{Cd}^{2+} / \mathrm{H}^{+}$有关 (Hirschi et al. , 1996)。在转基因烟草中表达证实 $C A X 1$ 与 $\mathrm{Ca}$ 的体 内平衡有关 (Hirschi，1999) , 而 $C A X 2$ 与 $\mathrm{Ca}^{2+} 、 \mathrm{Cd}^{2+}$ 、 $\mathrm{Mn}^{2+}$ 在体内的累积以及 $\mathrm{Mn}^{2+}$ 的耐性有关 (Hirschi et $a l$. 2000)。拟南芥中还发现了 CAX3、CAX4 基因， 它们在植物钙运转中的作用和其不同的 $\mathrm{N}$-末端有 关(Pittman et al. ,2002)。

\section{4 植物重金属耐性的其它机理}

除了以上的各种忍耐机制以外，植物还能通过 其它途径获得对重金属的忍耐能力。比如长期生活 在重金属浓度土壤上的植物能逐渐适应这种高浓度 的重金属胁迫，这可能受一小部分基因控制( Macnair ,1993)。植物中另一种重要的适应胁迫的机制 是诱导其它胁迫相关蛋白的表达, 如: 几丁质酶 (Chitinase)、热休克蛋白 (Heat shock proteins, HSP)、 富甘氨酸蛋白 (Glycine-rich proteins, GRP) 和致病相 关蛋白蛋白 (Pathogenesis related protein, PRP)等。柴 团耀等 (1998) 利用 $\mathrm{HgCl}_{2}$ 胁迫下的菜豆 (Phaseolus vulgaris L.cv. saxa)进行差别篮选叶片 cDNA 库, 分
离出 7 组不同的 $\mathrm{cDNA}$ 克隆重金属响应基因 PvSRI7 (Phaseolus vulgaris stress-related protein), $\mathrm{HgCl}_{2}$ 胁迫 可强烈地诱导 PvSR2 和 PR 蛋白基因的表达, 并能 提高 PR 蛋白、脱水素 (Dehydrin)、DnaJ-like 和 聚合 泛素( Polyubiquitin)的基因转录水平，这些蛋白质共 同作用与重金属抗性和体内平衡有关。PvSR2 在烟 草中表达表明它与植物 Cd 抗性相关 (Chai et al. , 2003)。作者进一步研究 DnaJ-like 蛋白表明它作为 HSP70 分子伴侣系统的组成部分, 可能在保护细胞 膜和酶蛋白的结构和功能等方面有重要作用 (Chai et al. 2000)。Neumann 等 (1994) 研究表明 HSP70 对 $\mathrm{Cd}$ 胁迫有响应，它的抗体位于细胞质和细胞核 中, 在细胞膜上也有分布, 它可能与膜抵抗 Cd 伤害 有关。

\section{5 结 语}

植物重金属耐性和耐性品种的选育一直是重金 属污染研究中的重要课题, 应用相关植物进行修复 重金属污染土地被认为是快速、经济和二次污染可 能性小的有效手段, 本文主要总结了近年来植物重 金属耐性机理特别是分子机理方面的主要进展。分 子生物学和生态学原理的结合, 为污染生态学的研 究开辟了另一个广阔的研究领域, 尽管目前这些研 究只是集中在少数的几种重金属 (如 $\mathrm{Cd} 、 \mathrm{Zn}$ ) 和少数 的植物 (主要是拟南芥和遏蓝菜等) 和酵母菌中, 相 关基因在植物基因工程中的应用也有待进一步研 究, 但是它所展示的前景无疑是值得期待的。近年 来、随着技术手段的进步和越来越多的相关蛋白质 和基因被分离鉴定出来,应用转基因等生物工程手 段 培育具有耐性和富集性特征的工程植物越来越 成为可能。尤其是把这种手段应用到近年来发现的 大量的超富集植物研究中，揭示其富集和忍耐的分 子机理, 进而发现可能在超富集植物中普遍存在的 高效的重金属结合物质及其控制基因，利用转基因 技术将其转移到生物量大、生长迅速的植物中，则会 大大提高重金属污染治理的效率和节约经济成本。 但是实现这一目的仍然有很多方面的工作需要进 行, 重金属耐性本身是植物生理系统共同作用的结 果, 目前的研究主要集中在几个主要过程中某一单 独的基因和蛋白质，如何使在某一过程占主导地位 的分子机理和其它分子过程协同作用, 有没有可能 使从吸收、转运、结合到分隔整个过程中各个基因共 同表达，从而获得较高的耐性和富集水平的植物都 值得进一步研究。 


\section{参 考 文 献}

Baker AJM (1981). Accumulators and excluders-strategies in the response of plant to heavy metals. Journal of Plant Nutrition, 3 , $643-654$.

Baker AJM, Brooks RR (1989). Terrestrial higher plants which hyperaccumulate metal elements - A review of their distribution, ecology, and photochemistry. Biorecovery, 1, 81-126.

Bernard C, Roosens N, Czernic P, Lebrun M, Verbruggen N (2004) . A novel CPx-ATPase from the cadmium hyperaccumulator Thlaspi caerulescens. FEBS Letters, 569, $140-148$.

Blo $\beta$ T, Clemens S, Nies DH (2002). Characterization of the ZAT1p zinc transporter from Arabidopsis thaliana in microbial model organisms and reconstituted proteoliposomes. Planta, $214,783-791$.

Cao H(曹慧), Han ZH(韩振海), Xu XF(许雪峰), Zhang Y(张 勇) (2002). Iron nutrition in higher plants. Plant Physiology Communications (植物生理学通讯), 38, 180 - 186. (in Chinese)

Chai TY (柴团耀), Zhang YX(张玉秀), Burkard G (1998). Heavy metal-responsive genes in Kidney bean: cloning of cDNA and gene expression analysis. Acta Phytophysiologica Sinica (植 物生理学报), 24, 399 - 404. (in Chinese with English abstract)

Chai TY, Zhang YX, Zhao WM (2000) . Cloning of cDNA and expression analysis of a DnaJ-like gene under heavy metal stress in bean. Progress in Natural Science, 2000, $198-203$.

Chai TY, Chen Q, Zhang YX, Dong J, An CC (2003) . Cadmium resistance in transgenic tobacco plants enhanced by expressing bean heavy metal-responsive gene PvSR2. Science in China: Series C-life sciences, 46, 623-630.

Chaney RL, Malik M, Li YM, Brown SL, Angle JS, Baker AJM (1997). Phytoremediation of soil metals. Current Opinions in Biotechnology, 8, 279-284 .

Clemens S, Kim EJ, Neumann D, Schroeder JI (1999). Tolerance to toxic metals by a gene family of phytochelatin synthases from plants and yeast. EMBO Journal, 18, 3325 - 3333 .

Clemens S (2001). Molecular mechanisms of plant metal tolerance and homeostasis. Planta, 212, $475-486$.

Cobbett CS (2000). Phytochelatins and their role in heavy metal detoxification. Plant Physiology, 123, 825-833.

Cobbett CS, Goldsbrough P (2002). Phytochelatins and metallothioneins, roles in heavy metal detoxification and homeostasis. Annual Review of Plant Biology, 53, 159 - 182.

Curie C, Alonso JM, Le Jean M, Ecker JR, Briat JF (2000). Involvement of NRAMP1 from Arabidopsis thaliana in iron transport. Biochemical Journal, 347, $749-755$.

Curie C, Briat JF (2003). Iron transport and signaling in plants. Annual Review of Plant Biology, 54, 183 - 206.

Delhaize E, Kataoka T, Hebb DM, White RG, Ryan PR (2003). Genes encoding proteins of the cation diffusion facilitator family that confer manganese tolerance. Plant Cell, 15, 1131 - 1142 .
Douchkov D, Gryczka C, Stephan UW, Hell R, Baumlein H (2005) . Ectopic expression of nicotianamine synthase genes results in improved iron accumulation and increased nickel tolerance in transgenic tobacco. Plant, Cell and Environment, 28, 365 374 .

Eide D, Broderius M, Fett J, Guerinot ML (1996) . A novel ironregulated metal transporter from plants identified by functional expression in yeast. Proceedings of the National Academy of Sciences of the United States of America, 93, 5624 - 5628.

Eren E, Argüello JM (2004) . Arabidopsis HMA2, a divalent heavy metal-transporting $\mathrm{P}_{\mathrm{IB}}$-Type ATPase, is involved in cytoplasmic $\mathrm{Zn}^{2+}$ homeostasis. Plant Physiology, 136, $3712-3723$.

Freeman JL, Persans MW, Nieman K, Albrecht C, Peer W, Pickering IJ, Salt DE (2004). Increased glu tathione biosynthesis plays a role in nickel tolerance in Thlaspi nickel hyperaccumulators. Plant Cell, 16, 2176-2191.

Galli U, Schüepp H, Brunold C (1994). Heavy metal binding by mycorrhizal fungi. Physiologia Plantarum, 92, $364-368$.

Garcia-Hernandez M, Murphy A, Taiz L (1998). Metallothioneins 1 and 2 have distinct but overlapping expression patterns in Arabidopsis. Plant Physiology, 118, $387-397$.

Gong JM, Lee DA, Schroeder JI (2003). Long-distance root-toshoot transport of phytochelatins and cadmium in Arabidopsis. Proceedings of the National Academy of Sciences of the United States of America, 100, 10118 - 10123.

Grill E, Winnacker EL, Zenk MH (1985). Phytochelatins: the principal heavy-metal complexing peptides of higher plants. Science, $230,674-676$.

Grill E, Loffler S, Winnacker EL, Zenk MH (1989). Phytochelatins, the heavy-metal-binding peptides of plants, are synthesized from glutathione by a specific $\gamma$-glutamylcysteine dipeptidyl transpeptidase (phytochelatin synthase). Proceedings of the National Academy of Sciences of the United States of America, 86, $6838-6842$.

Grotz N, Fox T, Connolly E, Park W, Guerinot ML, Eide D (1998). Identification of a family of zinc transporter genes from Arabidopsis that respond to zinc deficiency. Proceedings of the National Academy of Sciences of the United States of America, 95, $7220-7224$.

Ha SB, Smith AP, Howden R, Dietrich WM, Bugg S, OConnell MJ, Goldsbrough PB, Cobbett CS (1999). Phytochelatin synthase genes from Arabidopsis and the yeast Schizosaccharomyces pombe. Plant Cell, 11, 1153-1163.

Higuchi K, Suzuki K, Nakanishi H, Yamaguchi H, Nishizawa N K, Mori S (1999). Cloning of nicotianamine synthase genes, novel genes involved in the biosynthesis of phytosiderophores. Plant Physiology, 119, $471-480$.

Hirschi KD, Zhen RG, Cunningham KW, Rea PA, Fink GR (1996). CAX1, an $\mathrm{H}^{+} / \mathrm{Ca}^{2+}$ antiporter from Arabidopsis. Proceedings of the National Academy of Sciences of the United States of America, 93, 8782 - 8786. 
Hirschi KD (1999). Expression of Arabidopsis CAX1 in tobacco: altered calcium homeostasis and increased stress sensitivity. Plant Cell, 11, 2113 - 2122 .

Hirschi KD, Korenkov VD, Wilganowski NL, Wagner GJ (2000) . Expression of Arabidopsis CAX2 in tobacco. Altered metal accumulation and increased manganese tolerance. Plant Physiology, $124,125-133$.

Jasinski M, Ducos E, Martinoia E, Boutry M (2003). The ATPbinding cassette transporters: structure, function, and gene family comparison between rice and Arabidopsis. Plant Physiology, $131,1169-1177$.

Jentschke G, Goldbold DL (2000). Metal toxicity and ectomycorrhizas. Physiologia Plantarum, 107,107-116.

Kampfenkel K, Kushnir S, Babiychuk E, Inze D, van Montagu M (1995). Molecular characterization of a putative Arabidopsis thaliana copper transporter and its yeast homologue. Journal of Biological Chemistry, 270, 28479 - 28486.

Krämer U, Cotter-Howells JD, Charnock JM, Baker AJM, Smith AC (1996). Free histidine as a metal chelator in plants that accumulate nickel. Nature, 379, 635-638.

Lane BG, Kajioka R, Kennedy TD (1987). The wheat germ Ec protein is a zinc-containing metallothionein. Biochemical and Cell Biology, 65, 1001 - 1005 .

Lee J, Bae H, Jeong J, Lee JY, Yang YY, Hwang I, Martinoia E, Lee Y (2003). Functional expression of a bacteral heavy metal transporter in Arabidopsis enhances resistance to and decrease uptake of heavy metals. Plant Physiology, 133, 589 596.

Lee J, Shim D, Song WY, Hwang I, Lee Y (2004). Arabidopsis metallothioneins $2 \mathrm{a}$ and 3 enhance resistance to cadmium when expressed in Vicia faba guard cells. Plant Molecular Biology, $54,805-815$.

Li L, Kaplan J (1998) . Defects in the yeast high affinity iron transport system result in increased metal sensitivity because of the increased expression of transporters with a broad transition metal specificity. Journal of Biological Chemistry, 273, 22181 22187.

Li ZS, Lu YP, Zhen RG, Szczypka M, Thiele DJ, Rea PA (1997). A new pathway for vacuolar cadmium sequestration in Saccharomyces cerevisiae: YCF1-catalyzed transport of bis (glutathiona-to) cadmium. Proceedings of the National Academy of Sciences of the United States of America, 94, $42-47$.

Lu YP, Li ZS, Rea PA (1997). AtMRP1 gene of Arabidopsis encodes a glutathione S-conjugate pump: isolation and functional definition of a plant ATP-binding cassette transporter gene. Proceedings of the National Academy of Sciences of the United States of America, 94, 8243 - 8248 .

Lu YP, Li ZS, Drozdowicz YM, Hortensteiner S, Martinoia E, Rea PA (1998). AtMRP2, an Arabidopsis ATP binding cassette transporter able to transport glutathione S-conjugates and chlorophyll catabolites: functional comparisons with AtMRP1. Plant
Cell, 10, $267-282$.

Ma JF, Zheng SJ, Matsumoto H (1997a). Detoxifying aluminium with buckwheat. Nature, 390,569-570.

Ma JF, Ryan PR, Delhaize E (2001). Aluminium tolerance in plants and the complexing role of organic acids. Trends in Plant Science, 6, 273-278.

Ma M, Tsang WK, Lau PS, Wong YS (1997b). Cloning and sequencing of the metallothionein-like cDNA from Festuca rubra. Plant physiology, 114, 1136.

Macnair MR (1993). The genetics of metal tolerance in vascular plants. New Phytologist, 124, $541-559$.

Mench M, Martin E (1991). Mobilization of cadmium and other metals from two soils by root exudates of Zea mays L., Nicotiana tabacum L., Nicotiana rustica L. Plant and Soil, 132, 187 196.

Mizuno D, Higuchi K, Sakamoto T, Nakanishi H, Mori S, Nishizawa NK (2003). Three nicotianamine synthase genes isolated from maize are differentially regulated by iron nutritional status. Plant Physiology, 132, 1989 - 1997.

Nakanishi H, Yamaguchi H, Sasakuma T, Nishizawa NK, Mori S (2000) . Two dioxygenase genes, Ids3 and Ids2, from Hordeum vulgare are involved in the biosynthesis of mugineic acid family phytosiderophores. Plant Molecular Biology, 44, $199-207$.

Neumann D, Lichtenberger O, Günther D, Tschiersch K, Nover L (1994). Heat-shock proteins induce heavy-metal tolerance in higher plant. Planta, 194, 360-367.

Ortiz DF, Kreppel L, Speiser DM, Scheel G, McDonald G, Ow DW (1992). Heavy metal tolerance in the fission yeast requires an ATP-binding cassette-type vacuolar membrane transporter. EMBO Journal, 11, 3491 - 3499.

Ortiz DF, Ruscitti T, McCue KF, Ow DW (1995). Transport of metal-binding peptides by HMT1, a fission yeast ABC-type vacuolar membrane protein. Journal of Biological Chemistry, 270, $4721-4728$.

Papoyan A, Kochian LV ( 2004 ). Identification of Thlaspi caerulescens genes that may be involved in heavy metal hyperaccumulation and tolerance. Characterization of a novel heavy metal transporting ATPase. Plant Physiology, 136, 3814- 3823 .

Paulsen IT, Saier MH (1997). A novel family of ubiquitous heavy metal ion transport proteins. Journal of Membrane Biology, 156, $99-103$.

Pence NS, Larsen PB, Ebbs SD, Letham DL, Lasat MM, Garvin DF, Eide D, Kochian LV (2000). The molecular physiology of heavy metal transport in the $\mathrm{Zn} / \mathrm{Cd}$ hyperaccumulator Thlaspi caerulescens. Proceedings of the National Academy of Sciences of the United States of America, 97, 4956 - 4960.

Persans MW, Yan X, Patnoe JM, Krämer U, Salt DE (1999). Molecular dissection of the role of histidine in nickel hyperaccumulation in Thlaspi goesingense. Plant Physiology, 121, 1117 1126.

Persans MW, Nieman K, Salt DE (2001). Functional activity and 
role of cation-efflux family members in Ni hyperaccumulation in Thlaspi goesingense. Proceedings of the National Academy of Sciences of the United States of America, 98, 9995 - 10000.

Pittman JK, Sreevidya CS, Shigaki T, Ueoka-Nakanishi H, Hirschi KD (2002) . Distinct N-terminal regulatory domains of $\mathrm{Ca}^{2+} / \mathrm{H}^{+}$ antiporters. Plant Physiology, 130, $1054-1062$.

Rauser WE (1999). Structure and function of metal chelators produced by plants. Cell Biochemistry and Biophysics, 31, $19-48$. Rauser WE (1995). Phytochelatins and related peptides: structure, biosynthesis, and function. Plant Physiology, 109, 1141 1149 .

Robinson NJ, Wilson JR, Turner JS ( 1996). Expression of the type2 metallothionein-like gene MT2 from Arabidopsis thaliana in $\mathrm{Zn}^{2+}$-metallothionein-deficient Synechococcus PCC 7942: putative role for MT2 in $\mathrm{Zn}^{2+}$ metabolism. Plant Molecular Biology, $30,1169-1179$.

Salt DE, Wagner GJ (1993) . Cadmium transport across tonoplast of vesicles from oat roots: evidence for a $\mathrm{Cd}^{2+} / \mathrm{H}^{+}$antiport activity. Journal of Biological Chemistry, 268, 12297 - 12302 .

Salt DE, Rauser WE (1995). MgATP-dependent transport of phytochelatins across the tonoplast of oat roots. Plant Physiology, $107,1293-1301$.

Salt DE (2001). Responses and adaptations of plants to metal stress. In: Hawkesford MJ, Buchaer P eds. Molecular Analysis of Plant Adaptation to the Environment. Kluwer academic publishers, Dordrecht, 159 - 177.

Song WY, Sohn EJ, Martinoia E, Lee YJ, Yang YY, Jasinski M, Forestier C, Hwang I, Lee Y (2003). Engineering tolerance and accumulation of lead and cadmium in transgenic plants. Nature Biotechnology, 21, $914-919$.

Steffens JC, Hunt DF, Williams BG (1986) . Accumulation of nonprotein metal-binding polypeptides ( $\gamma$-Glu-Cys) n-Gly in selected cadmium-resistant tomato cells. Journal of Biological Chemistry, 261,13879 - 13882 .

Stephan UW, Scholz G (1993). Nicotianamine: mediator of transport of ion and heavy metals in the phloem? Physiologia Plantarum, 88, 522-527.

Takahashi M, Yamaguchi H, Nakanishi H, Shioiri T, Nishizawa N
K, Mori S (1999) . Cloning two genes for nicotianamine aminotransferase, a critical enzyme in iron acquisition (strategy ii) in graminaceous plants. Plant Physiology, 121, 947 - 956.

Thomine S, Wang R, Ward JM, Crawford NM, Schroeder JI (2000) . Cadmium and iron transport by members of a plant metal transporter family in Arabidopsis with homology to Nramp genes. Proceedings of the National Academy of Sciences of the United States of America, 97, 4991 - 4996.

Vallino M, Drogo V, Abba S, Perotto S (2005). Gene expression of the ericoid mycorrhizal fungus Oidiodendron maius in the presence of high zinc concentrations. Mycorrhiza, 15,333-344.

van der Zaal BJ, Neuteboom LW, Pinas JE, Chardonnens AN, Schat H, Verkleij JAC, Hooykaas PJJ (1999). Overexpression of a novel Arabidopsis gene related to putative zinc-transporter genes from animals can lead to enhanced zinc resistance and accumulation. Plant Physiology, 119, 1047 - 1055 .

Verkleij JAC, Koevoets PLM, Blake-Kalff MMA, Chardonnens AN (1998). Evidence for an important role of the tonoplast in the mechanism of naturally selected Zn tolerance in Silene vulgaris. Journal of Plant Physiology, 153, 188 - 191 .

Verret F, Gravot A, Auroy P, Preveral S, Forestier C, Vavasseur A, Richaud P (2005). Heavy metal transport by AtHMA4 involves the N-terminal degenerated metal binding domain and the C-terminal His (11) stretch. FEBS Letters, 579, 1515 - 1522.

Zhao H, Eide D (1996a). The yeast ZRTI gene encodes the zinc transporter protein of a high-affinity uptake system induced by zinc limitation. Proceedings of the National Academy of Sciences of the United States of America, 93, 2454 - 2458.

Zhao H, Eide D (1996b). The ZRT2 gene encodes the low affinity zinc-transporter in Saccharomyces cerevisiae. Journal of Biological Chemistry, 271, $23203-23210$.

Zhao ZQ(赵中秋), Cui YJ(崔玉静), Zhu YG(朱永官)（2003）. Effect of mycorrhiza and roots exudates on resistance of plants to heavy metals. Chinese Journal of Ecology (生态学杂志), 22 (1) , 81-84. (in Chinese with English abstract)

Zhou J, Goldsbrough PB (1994). Functional homologs of fungal metallothionein genes from Arabidopsis. Plant Cell, 6, 875 884 . 